\title{
A finger or not? Prostate examinations by non-urologists at a South African academic institution
}

\author{
K Spencer, MB BCh \\ Department of Urology, Faculty of Health Sciences, University of the Witwatersrand, Johannesburg, South Africa
}

Corresponding author: K Spencer(doctorkspencer@gmail.com)

Background. According to the South African (SA) National Cancer Registry 2009, prostate cancer is the most commonly diagnosed solidorgan cancer in SA men: the incidence increases at 3\% every year, with 1/26 men developing prostate cancer in their lifetime and five men dying every day. Screening for prostate cancer by performing a digital rectal examination (DRE) adds to the sensitivity and specificity of serum prostate-specific antigen (PSA) testing and is an important component of the assessment for the early detection of prostate cancer. Objectives. To ascertain whether doctors who are not in the field of urology perform DREs and PSA testing to screen for prostate cancer, where indicated, and to determine the reason, if any, why doctors do not perform prostate examinations.

Methods. Doctors (including specialists and primary care practitioners) who consulted adult male patients in the emergency departments and polyclinics of three academic hospitals were included in the sample and asked to complete an anonymous questionnaire. Doctors with specialist urological training were excluded from the sample. The questionnaire included the following aspects: gender, year of graduation and university, current position, knowledge of PSA screening and DRE, whether these were being performed, and reasons for nonperformance.

Results. The response rate was 303/350 participants. Fifty-nine percent of the respondents were female. The median duration of experience was 5 years. The mean (standard deviation) knowledge score with regard to screening was $56.1 \%$ (20). The PSA test alone was most frequently done (35\%), followed by asking the patient about urinary tract symptoms (28\%). In contrast, DRE of the prostate, alone or combined with a PSA test, was performed less frequently ( $13.2 \%$ and $10.6 \%$, respectively). Some of the most common reasons for not performing a DRE included: 'It's more convenient to do a PSA test'; 'Urologists will examine the prostate anyway'; 'No privacy in the emergency department/admission ward'; 'Prostate exam is not relevant to my practice'; 'There is not enough time'; and 'The practitioner forgets'. Doctors with $>7$ years of working experience are the least likely to do a DRE.

Conclusion. Performance rates of DRE and PSA testing by doctors who are not urologists are very low, which may have significant clinical implications. It is recommended that SA prostate cancer screening guidelines are necessary to change practice with regard to this condition.

S Afr Med J 2017;107(7):631-635. DOI:10.7196/SAMJ.2017.v107i7.12208

According to the South African (SA) National Cancer Registry 2009, prostate cancer is the most commonly diagnosed solid-organ cancer in SA men. ${ }^{[1]}$ The incidence of prostate cancer in SA increases at $~ 3 \%$ every year and 1/26 men are at risk of developing cancer in their lifetime ${ }^{[1]}$ Five men die of prostate cancer in SA every day. ${ }^{[1]}$

The American Urology Association guidelines recommend individualised screening for prostate cancer in men $<55$ years of age, based on high risk (positive family history or African American race) ${ }^{[2]}$ For men aged 55 - 69 years, they recommend shared decision-making based on values and preferences. ${ }^{[2]}$ The US Preventive Services Task Force has a grade D recommendation against prostate cancer screening, but concerns have recently been raised that these cancers may be of a higher grade and stage on subsequent detection. ${ }^{[3,4]}$

Screening for prostate cancer in SA public hospital settings entails a digital rectal examination (DRE) and serum prostatespecific antigen (PSA) testing. Most prostate cancers are located in the peripheral zone of the prostate and may be detected by a DRE when the volume is $\geq 0.2 \mathrm{~mL} .{ }^{[5]} \mathrm{In} \sim 18 \%$ of all patients, prostate cancer is detected by a suspect DRE alone, irrespective of the serum PSA level. ${ }^{[5]} \mathrm{An}$ abnormal DRE is associated with an increased risk of a higher Gleason score and should therefore be considered an indication for prostate biopsy. ${ }^{[5]}$ A PSA level $>4 \mathrm{ng} / \mathrm{mL}$ is considered abnormal. Each test (DRE and PSA) identifies a proportion of cancers, with greater rates of detection when used in combination. A positive finding on DRE when combined with an elevated PSA level increases the positive predictive value of detecting cancer on prostate biopsy by $26.2 \%$ compared with an elevated PSA level but a normal prostate on DRE. ${ }^{[6]}$

Some studies have found the DRE to be of limited value. The Prostate, Lung, Colorectal and Ovarian Cancer Screening trial found no long-term benefit with DRE and PSA testing. A suspect DRE in patients with a PSA level $\leq 2 \mathrm{ng} / \mathrm{mL}$ has a positive predictive value of $5-30 \%{ }^{[5]}$ DRE has a sensitivity of $55-68 \%$ in asymptomatic men, but values as low as $18-22 \%$ have been reported. ${ }^{[7]}$ Bretton $^{[8]}$ found that of $8 \%(n=60)$ of men with abnormal DRE findings and a normal PSA, only $3 \%(n=2)$ had prostate cancer.

DRE could prove beneficial in detecting other pathological conditions, e.g. occult blood in the stool suggestive of colon or rectal cancer, other colorectal pathology, and benign prostatic hyperplasia.

There is a high concordance of abnormal DRE results between general practitioners and urologists, which supports the finding that general practitioners or other non-urologists should always perform DREs as part of their examination for the early detection of cancer. ${ }^{[9]}$ However, such doctors may not have the time to do routine screening owing to large patient loads and minimal time allowed with each patient. In overcrowded casualty settings, doctors may also not have the appropriate privacy for intimate examinations. 
The objective of this study was therefore to ascertain whether SA doctors who are not in the field of urology perform DREs to assess the prostate for cancer, where indicated. The study also aimed to determine the reason why doctors do not perform prostate examinations at the academic hospitals, as mentioned below.

\section{Methods \\ Sample size}

Sample size was calculated using estimates of $50 \%$, with a $5 \%$ precision and a $95 \%$ confidence level. A sample size of 385 was required.

\section{Study participants}

The duration of experience was estimated as the number of years between graduation and the beginning of 2016, as the survey was done in the first 6 months of 2016. The doctor had to consult adult male patients; specialists and primary care practitioners working in emergency departments and polyclinics were also included. Doctors with specialist urological training were not part of the sample. Doctors from three University of the Witwatersrand academic hospitals were included: Charlotte Maxeke Johannesburg Academic Hospital, Helen Joseph Hospital, and Chris Hani Baragwanath Hospital.

Permission was obtained from the dean of the Faculty of Health Sciences and the management at each hospital. Ethics approval was obtained from the Human Research Ethics Committee at the University of the Witwatersrand (ref. no. M151102).

\section{Data collection process/tool}

Doctors were approached by the researcher and given an information sheet before an academic meeting. The participants completed an anonymous questionnaire that was divided into four domains: the respondent's demographics; knowledge of prostate cancer assessment; practice of DRE and PSA testing; and reasons for non-performance of a DRE. Completing the questionnaire was voluntary and considered to presume consent. Doctors' names and the hospitals were not recorded on the questionnaire to maintain anonymity. The knowledge score was calculated by scoring the responses as 1 for each correct answer to each of the 8 items in questions $6-8$, thus creating a score out of 8 (Table 1 ). This was then converted to a percentage.

The questionnaire was based on similar questionnaires from other studies. ${ }^{[10-12]}$ It was modified to the SA context by adding more reasons for non-performance of the DRE, such as no lubrication jelly and no space to examine the patient in the admission ward. The questionnaire was then piloted on a small cohort of doctors to check for validity of the modifications. The completed forms were placed in a sealed box in an administrator's office to further protect the respondents' identities. A total of 350 questionnaires were handed out.

\section{Statistical analysis}

Descriptive analysis of the data was carried out as follows: categorical variables were summarised by frequency and percentage tabulation and illustrated by bar charts. Continuous variables were summarised by the mean, standard deviation (SD), median and interquartile range (IQR), and their distribution was illustrated by histograms.

The $\chi^{2}$ test was used to assess the relationship between categorical study variables and gender, duration of experience, undergraduate university and field of practice. Fisher's exact test was used for $2 \times 2$ tables or where the requirements for the $\chi^{2}$ test could not be met. The strength of the associations was measured by Cramér's V and the phi coefficient, respectively.

The relationship between knowledge score and gender, duration of experience, undergraduate university, field of practice and practice was assessed by the $t$-test (or analysis of variance (ANOVA) for more than two categories). The strength of the associations was measured by Cohen's $d$ for parametric tests and the $r$-value for the non-parametric tests.

Data were analysed using SAS 9.4 (SAS Institute Inc., USA) (5\% significance level).

\section{Results}

The response rate was 303/350 questionnaires completed $(86.6 \%)$. The majority of the respondents were female $(59.1 \%)$. The median duration of experience was 5 years (IQR 2 - 9 years; range 0 - 48 years). Of

Table 1. Sample of questionnaire: Knowledge score - Questions 6 - 8

\begin{tabular}{lll}
\hline Question & Response & Ch \\
\hline $\begin{array}{l}\text { 6. As far as you know, at what age should } \\
\text { one start screening a patient for prostate } \\
\text { cancer? }\end{array}$ & \\
$\qquad \begin{array}{ll}\text { Correct answer } \rightarrow \\
\text { 45 years }\end{array}$ & 1 \\
& 40 years & 2 \\
& 45 years & 3 \\
& 50 years & 4 \\
& 55 years & 5
\end{tabular}

7. What is considered to be the level above which the prostate-specific antigen (PSA) level is interpreted as abnormal?

$1 \mathrm{ng} / \mathrm{mL}$
$2 \mathrm{ng} / \mathrm{mL}$
$4 \mathrm{ng} / \mathrm{mL}$
$6 \mathrm{ng} / \mathrm{mL}$

8. Please indicate whether you agree or disagree with each of the following statements:

\begin{tabular}{|c|c|c|c|}
\hline $\begin{array}{l}\text { A. A doctor should do PSA testing before } \\
\text { doing the prostate examination }\end{array}$ & 1 & 2 & 2 \\
\hline $\begin{array}{l}\text { B. A prostate examination falsely elevates } \\
\text { the PSA }\end{array}$ & 1 & 2 & 2 \\
\hline $\begin{array}{l}\text { C. Catheterisation gives a false-positive } \\
\text { PSA result }\end{array}$ & 1 & 2 & 1 \\
\hline $\begin{array}{l}\text { D. Obstructive uropathy gives a false- } \\
\text { positive PSA result }\end{array}$ & 1 & 2 & 1 \\
\hline $\begin{array}{l}\text { E. Prostatitis gives a false-positive PSA } \\
\text { result }\end{array}$ & 1 & 2 & 1 \\
\hline F. Level of the PSA is age dependent & 1 & 2 & 1 \\
\hline
\end{tabular}


all national universities, $45.9 \%$ of doctors had been trained at the University of the Witwatersrand. The two largest groups of respondents were interns (35.3\%) and registrars (26.7\%). Respondents came predominantly from the field of internal medicine (47.9\%), followed by primary care practitioners $(16.6 \%)$, general surgeons $(16 \%)$, surgical subspecialties (13.5\%) and other (10\%). None of them had specialist urology training.

The mean (SD) knowledge score was $56.1 \%(20)$, with a range of $0-100 \%$. Each component of the knowledge questionnaire was analysed. With regard to age for initiating prostate cancer screening, $40.3 \%$ recommended 40 years, $30 \%$ suggested 50 years, and $17.8 \%$ recommended 45 years (Fig. 1).

The respondents' knowledge of the causes of a false-positive PSA reading is represented in Fig. 2. Sixty-six percent of respondents thought that the PSA level was age dependent, with $69.6 \%$ predicting that the normal value was $>4 \mathrm{ng} / \mathrm{mL}$.

The next four questions pertained to the doctors' frequency of practices. Across all four items, the frequency of practices (as measured by answers of 'always' and 'almost always') appears to be low. Of the four practices, the PSA test alone is practised most frequently (35.0\%), followed by asking the patient about urinary tract symptoms (27.7\%). In contrast, a prostate examination, presumably by DRE, on its own, or in conjunction with a PSA test, is done less frequently $(13.2 \%$ and $10.6 \%$, respectively) (Fig. 3).

A DRE is not routinely done by $25.7 \%$ of doctors. No significant gender difference was noted $(p=0.66)$. With regard to PSA testing, $20.1 \%$ never, $44.9 \%$ sometimes and $35.0 \%$ always/almost always perform a PSA test. A combination of PSA testing and a prostate examination is never performed by $27.7 \%$ of doctors, while $61.7 \%$ sometimes perform both, and only $10.6 \%$ perform both.

The next set of questions explored the reasons why doctors do not perform a prostate examination (Fig. 4).

Qualitative analysis revealed the following reasons for not performing the prostate examination: performance of examination only where indicated; the doctor not being good at preventive medicine; laboratory gate-keeping system blocking the doctor from doing PSA testing; only enough time for the doctor to focus on the acute complaint; it may pass as sexual harassment; psychiatry female doctors don't do DREs; the doctor's finger is too short to reach the prostate; low confidence to adequately assess the prostate; the DRE is not relevant to the doctor's practice; and the doctor only does a DRE if the patient is symptomatic.

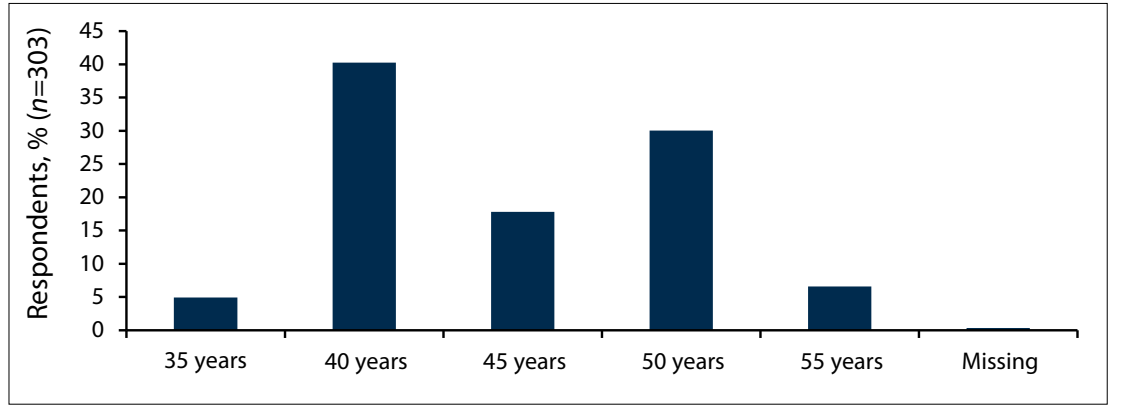

Fig. 1. Recommended prostate cancer screening age.

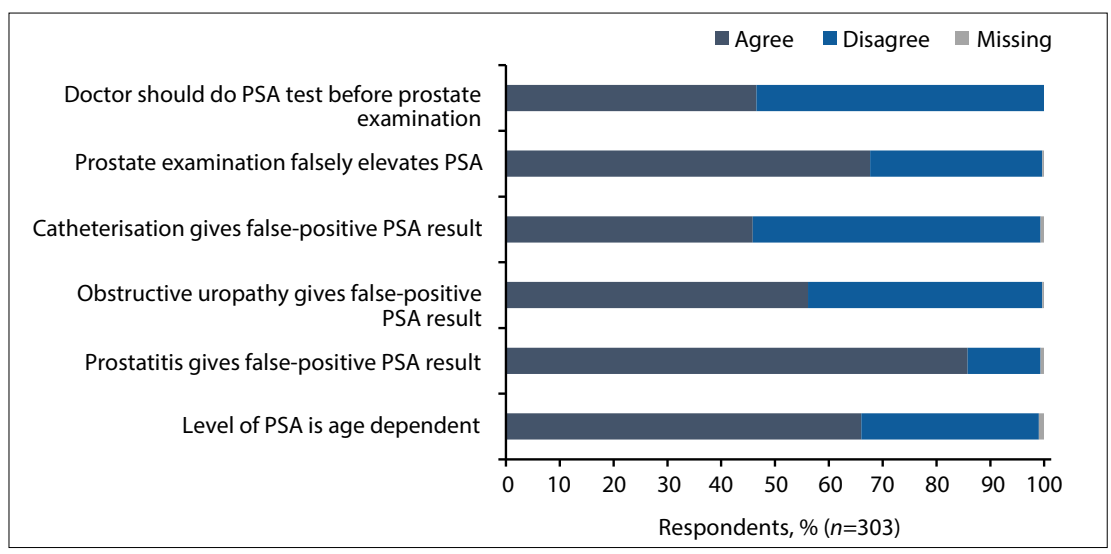

Fig. 2. Aetiology of false-positive PSA levels (question 8). (PSA = prostate-specific antigen.)

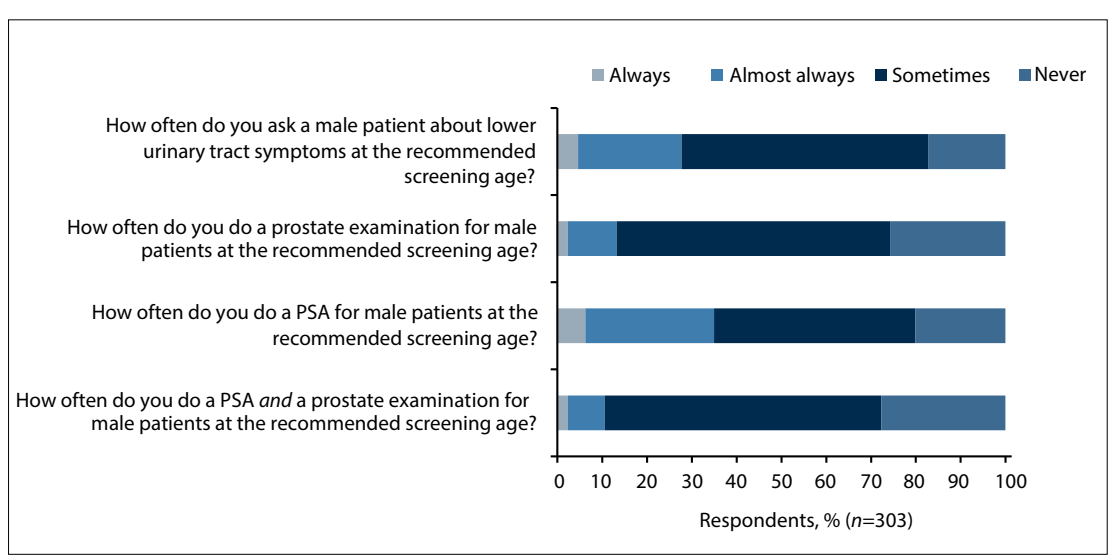

Fig. 3. Frequency of respondents' prostate cancer screening practice. (PSA = prostate-specific antigen.)

There were significant, weak associations between gender, and four reasons for not performing the prostate examination. In all cases, these reasons were more prevalent for women than for the men. These reasons included no privacy for examination $(p=0.0014)$; cultural or religious convictions $(p=0.013)$; anticipated patient refusal ( $p=0.0002)$; and concern about the patient's modesty ( $p=0.035$; Cramér's V 0.15 - 0.24).

There was a significant, weak association between duration of experience and the practice of performing a prostate examination (only) for male patients at the recommended screening age ( $\chi^{2}$ test; $p=0.014$; Cramér's V 0.18). The trend is not very clear, but it appears that those who never do the prostrate examination are predominantly in the $>7$-year experience categories.

There were significant, weak associations between field of practice and all four practice items ( $\chi^{2}$ test; Cramér's V 0.20 - 0.27) (Fig. 5). With regard to enquiring about symptoms, this practice was less prevalent in general surgery and the surgical subspecialties compared with all other areas $(p=0.001)$. General surgeons were more likely to perform the prostate examination only $(p<0.0001)$ as opposed to physicians, who were more likely to do only PSA testing $(p<0.0001)$. Primary care practitioners, general surgeons and surgical subspecialists were more likely to do both the DRE and PSA testing $(p=0.010)$. 


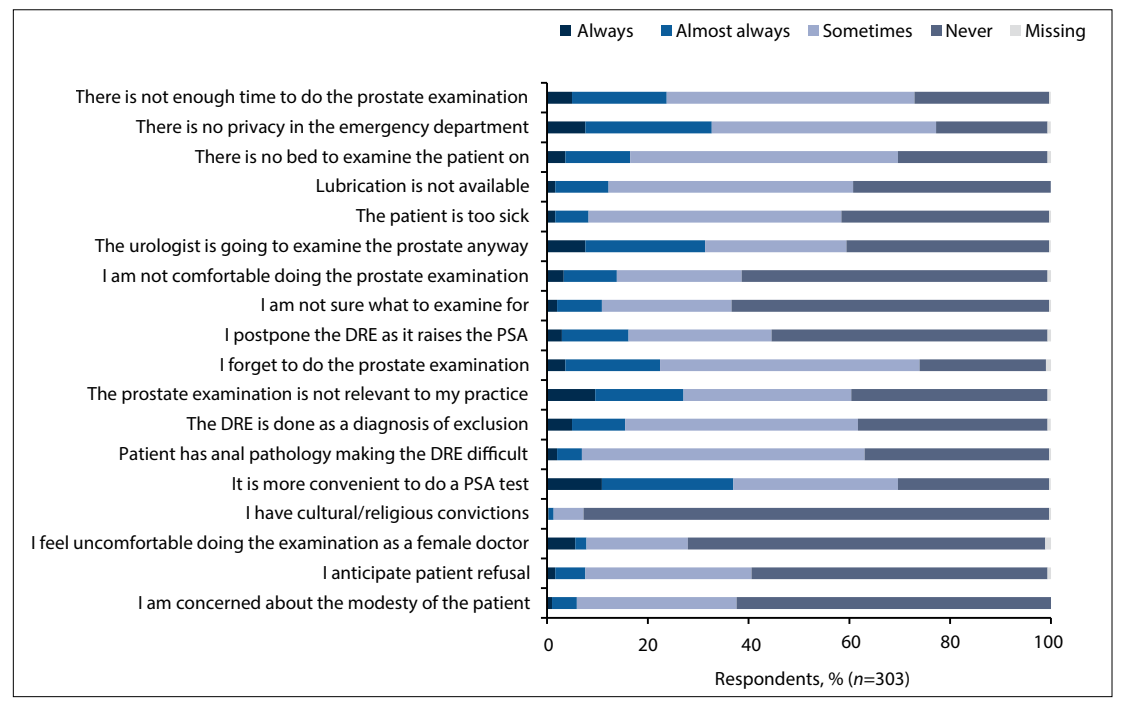

Fig. 4. Reasons of non-urologists for not performing a digital rectal examination. (DRE = digital rectal examination; $P S A=$ prostate-specific antigen.)

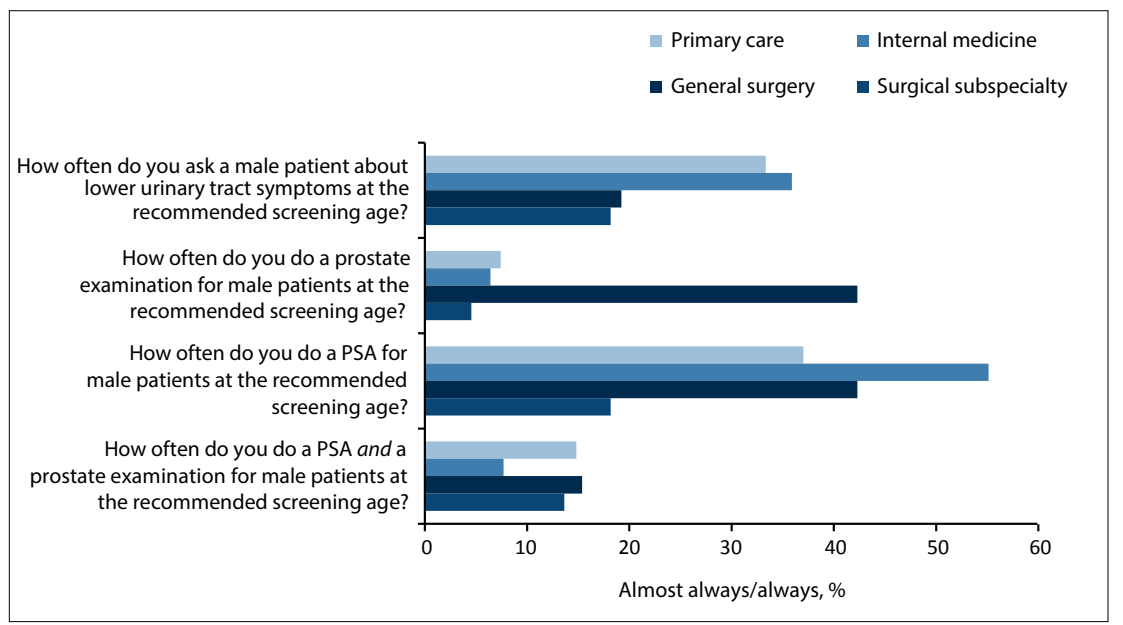

Fig. 5. Frequency of prostate cancer screening per medical discipline. (PSA = prostate-specific antigen.)

There was no significant association between knowledge score and practice items or reasons for not performing the prostate examination.

\section{Discussion}

Despite the importance of screening for prostate cancer, this practice still remains low among non-urologists. The age at which to start screening for prostate cancer is controversial, especially without guidelines in place. Blacks are the largest racial group in SA, and are also at higher risk of prostate cancer. An SA study found that men aged $<50$ years compared with those $>50$ years had a significantly greater proportion of poorly differentiated adenocarcinoma (53\%), locally advanced (stage T3 - 4) tumours (56\%), haematogenous metastases (75\%), significantly higher serum PSA levels at diagnosis (median $74 \mathrm{ng} / \mathrm{mL}$ ) and shorter survival. ${ }^{[13]}$ General consensus therefore dictates that
In this study it was found that $25.7 \%$ of doctors do not perform a DRE routinely. This is in contrast to a study by Lim and Quinlan, ${ }^{[18]}$ who found that $25 \%$ of patients had undergone a DRE. Leff et al. ${ }^{[19]}$ found that $41 \%$ of doctors reported that they regularly performed annual DREs on outpatients. For inpatients, $29 \%$ perform it as part of every consultation, while $56 \%$ perform it only when clinically indicated. ${ }^{[19]}$ They also showed that of the 85 charts analysed, $56 \%$ did not document a DRE. ${ }^{[19]}$ Turner and Brewster ${ }^{[11]}$ found that house officers performed DREs frequently; $53 \%$ had performed $>50$ DREs and only $15 \%$ had performed $<30$ DREs. Findings were infrequently confirmed by a colleague who had also examined the patient; $37 \%$ of respondents indicated that their findings were hardly ever confirmed, and $47 \%$ mentioned that their DRE findings were occasionally confirmed. ${ }^{[11]}$ Freeman $^{[14]}$ found that for $>80 \%$ of patients admitted to a medical teaching unit there was no documentation of a DRE.

Regarding PSA testing in this study, 35.1\% of respondents always perform a PSA test. Lim and Quinlan ${ }^{[18]}$ found that of 89 patients, $45 \%$ had PSA test results. Firzara and $\mathrm{Ng}^{[15]}$ found that most $(94.9 \%)$ of their respondents would perform a PSA test if they intended to screen for prostate cancer.

In the current study, a combination of PSA testing and prostate examination is also infrequently performed, with $27.7 \%$ never, $61.7 \%$ sometimes, and $10.6 \%$ always/almost always doing both. Lim and Quinlan ${ }^{[18]}$ also reported poor performance rates, with $17 \%$ of patients having both a DRE and PSA testing. This is in contrast to Pendleton et al., ${ }^{[10]}$ who found that $79 \%$ of patients had both PSA and DRE findings. McNaughton et al. ${ }^{[17]}$ indicated that nearly all (97\%) of the respondents agreed that performing both the PSA test and a DRE is more effective in assessing the presence of prostate cancer.

In our setting we therefore have to ask why there is such poor performance of this practice. Is gender a determining factor? In this study there was no significant correlation with gender. Leff et al. ${ }^{[19]}$ found no correlation with gender of either the patient or resident with regard to comfort level. In two separate studies it was found that female doctors performed less than half the number of DREs than their male counterparts. ${ }^{[12,14]}$

Another consideration is years of experience since graduation. This study found that those who never perform DREs are predominantly in the $>7$-year experience category. This may be because many doctors become 
more experienced and select patients on whom to perform a DRE. It could also be that with experience they do not find it useful and would rather use PSA testing. Wong et al.$^{[12]}$ found that the number of DREs correlated $(r=0.37 ; p<0.01)$ with the number of years of clinical experience, ranging from an average of 29 DREs among physicians within 4 years post graduation to an average of 123 DREs per year among physicians who had been practising for $>20$ years. Turner and Brewster ${ }^{[11]}$ found that, despite the additional experience gained since graduation, exposure to pathology remained limited; $68 \%$ of house officers had palpated $<5$ clinically malignant prostate glands.

With regard to medical disciplines, we found that general surgeons followed by primary care physicians and internal medicine physicians were more likely to assess the prostate than other surgical subspecialties. General surgeons are more likely to perform a rectal examination to assess for colorectal pathology and include examination of the prostate simultaneously. Lim and Quinlan ${ }^{[18]}$ noted that their medical teams were more likely not to perform a DRE $(78.3 \%)$ than were the surgical teams (67.7\%). They also noted that more medical patients had had PSA testing than had surgical patients (48.1\% v. 39.9\%). Wong et $a l .{ }^{[12]}$ noted that fewer of the gastrointestinal fellows would assess the prostate compared with primary care and general physicians.

In this study, $60.7 \%$ would never, $24.8 \%$ would sometimes, and $13.9 \%$ would always/almost always not perform a DRE because they felt uncomfortable. Turner and Brewster ${ }^{[11]}$ found that $16 \%$ of doctors at the end of their house year were not at all confident about their ability to give an opinion based on their DRE findings, $81 \%$ were reasonably confident, and $2 \%$ were very confident.

Finally, the doctors were asked why they do not perform DREs. The reasons given in this study were very similar to those given in other studies. Hennigan et al. ${ }^{[20]}$ noted that doctors may be embarrassed, a patient may refuse, or a chaperone may not be available. One doctor stated: 'I feel quite strongly that in the absence of feedback it is an intrusive waste of time carrying out rectal examinations.'[20] Wong et al. ${ }^{[12]}$ noted that the lack of comfort may be explained by several factors, including inadequate training and physician reluctance to perform a rectal examination on a patient, particularly of the opposite gender. Respondents with less experience were more likely to cite perceived invasiveness, patient modesty, and anticipated patient refusal as reasons for not performing a DRE. ${ }^{[12]}$ Other reasons cited for not performing a DRE included cultural-religious convictions, too much trouble, age of patient, limited value/outdated/not useful, and too invasive as a routine examination. Leff et al. ${ }^{[19]}$ noted that $13 \%$ perform the examination for fear of legal liability or because they are told to do so. Forty-six percent did not perform the examination, as they felt it would not provide useful information. ${ }^{[19]}$

The underperformance of the DRE not only leads to non-detection of some prostate cancer cases, but also deprives junior doctors of training. ${ }^{[18]}$ Students should start with lectures on the theoretical aspects of performing a rectal examination, followed by practical tutorials, using aids such as a pelvic trainer. ${ }^{[12]}$ Teaching the physical examination on real patients remains an important part of medical education, and supervised performance of the DRE in wards and clinics by tutors and senior clinicians is still advocated; this should continue during postgraduate training. ${ }^{[12]}$

\section{Study limitations}

The preferred sample for this population sample should have been 385 doctors. The actual sample size of 303 corresponds to a precision of 5.6\% (rather than 5.0\%), which is, however, acceptable. Only
Johannesburg hospitals were included owing to time and resource constraints, as the author is a registrar at the Wits academic complex and was unable to travel to obtain more samples.

\section{Conclusion}

Performance rates of the DRE at our academic institution by doctors who are not urologists are very low. This may have significant clinical implications. It is recommended that SA prostate cancer screening guidelines are needed to institute change.

Acknowledgements. I would like to thank Petra Gaylard for assistance with the statistical analysis and Dr Alison Bentley for assistance with the protocol preparation and writing of the article.

Author contributions. KS: research concept, data collection, data analysis and interpretation, and drafting of the article.

Funding. Self-funded.

Conflicts of interest. None.

1. National Cancer Registry 2009. http://www.cansa.org.za/files/2015/03/NCR_2009_FINAL.pdf (accessed 16 November 2016).

2. Carter HB, Albertsen PC, Barry MJ, et al. Early detection of prostate cancer: American Urological Arter HB, Albertsen PC, Barry M), et al. Early detection of prostate cancer: American Urotion guideline. J Urol 2013;190(2):419-426. https://doi.org/10.1016/j.juro.2013.04.119
Assocition

3. Moyer VA. Screening for prostate cancer: US Preventive Services Task Force recommendation statement. Ann Intern Med 2012;157(2):120-134. https://doi.org/10.7326\%2F0003-4819-149-3-200808050-00008 4. Fleshner K, Carlsson SV, Roobol MJ. The effect of the USPSTF PSA screening recommendation on prostate cancer incidence patterns in the USA. Nature Rev Urol 2017;14:26-37. https://do org/10.1038\%2Fnrurol.2016.251

5. Mottet N, Bastian PJ, Bellmunt J, et al. European Association of Urology (EAU) Guidelines Group for Prostate Cancer: Prostate Cancer Update. 2014. http://uroweb.org/wp-content/uploads/09-ProstateCancer_LR.pdf (accessed 16 November 2016).

6. Gosselaar C, Roobol MJ, Roemeling S, Schroëder FH. The role of the digital rectal examination in subsequent screening visits in the European randomized study of screening for prostate cancer (ERSPC), Rotterdam. Eur Urol 2008b;54(3):581-588. https://doi.org/10.1016\%2Fj.eururo.2008.03.104 7. Carvalhal GF, Smith DS, Mager DE, Ramos C, Catalona WJ. Digital rectal examination for detecting prostate cancer at prostate specific antigen levels of $4 \mathrm{ng} / \mathrm{ml}$ or less. J Urol 1999;161(3):835-839. https:// doi.org/10.1016\%2Fs $0022-5347 \% 2801 \% 2961785-3$

8. Bretton PR. Prostate-specific antigen and digital rectal examination in screening for prostate cancer: A community-based study. South Med J 1994;87(7):720-723. https://doi.org/10.1097\% 2F00007611-199407000-00009

9. Walsh AL, Considine SW, Thomas AZ, Lynch TH, Manecksha RP. Digital rectal examination in Walsh AL, Considine $\mathrm{SW}$, Thomas $\mathrm{AZ}$, Lynch $\mathrm{TH}$, Manecksha RP. Digital rectal examination in
primary care is important for early detection of prostate cancer: A retrospective cohort analysis study. Br J Gen Pract 2014;64(629):783-787. https://doi.org/10.3399\%2Fbjgp14x682861

10. Pendleton J, Curry RW, Kaserian A, et al. Knowledge and attitudes of primary care physicians regarding prostate cancer screening. J Natl Med Assoc 2008;100(6):666-670. https://doi.org/10.1016 $\% 2$ Fs $0027-9684 \% 2815 \% 2931339-0$

11. Turner KJ, Brewster SF. Rectal examination and urethral catheterization by medical students and house officers: Taught but not used. BJU lnt 2000;86(4):422-426. https://doi.org/10.1046\%2Fj.1464410x.2000.00859.x

12. Wong RK, Drossman DA, Bharucha AE, et al. The digital rectal examination: A multicenter surver of physicians' and students' perceptions and practice patterns. Am J Gastroenterol 2012;107(8):11571163. https://doi.org/10.1038/ajg.2012.23

13. Heyns CF, Fisher M, Lecuona A, van der Merwe A. Should baseline PSA testing be performed in men aged 40 to detect those aged 50 or less who are at risk of aggressive prostate cancer? S Afr Med J 2011;101(9):642-644.

14. Freeman HJ. Documentation of rectal examination performance in the clinical teaching unit of a university hospital. Can J Gastroenterol Hepatol 2000;14(4):272-276. https://doi.org/10.1155\%2F $2000 \% 2 \mathrm{~F} 297390$

15. Firzara AMT, Ng CJ. Knowledge and practice of prostate cancer screening among general practitioners . Firzara AMT, $\mathrm{Ng}$ CJ. Knowledge and practice of prostate cancer screening among general practitioners
in Malaysia: A cross-sectional study. BMJ Open 2016;6(9):e011467. https://doi.org/10.1136\%2Fbmjo pen-2016-011467

16. Johnson K, Chang M, Sun Y, Miyake M, Rosser CJ. Attitudes and knowledge of primary care physicians regarding prostate cancer screening. J Cancer Educ 2013;28(4):679-683. https://doi org/10.1007\%2Fs13187-013-0533-6

17. McNaughton D, Aiken W, McGrowder D. Factors affecting prostate cancer screening behaviour in a discrete population of doctors at the University Hospital of the West Indies, Jamaica. Asian Pac J Cancer Prev 2011;12(5):1201-1205.

18. Lim CH, Quinlan DM. Are doctors examining prostates in university hospitals? Urology 2007;70 (5):843845. https://doi.org/10.1016\%2Fj.urology.2007.07.010

19. Leff DB, Azzouz M, Molloy PJ, Kania RJ. Attitudes toward vs performance of rectal exams. Gastroenterology 1997;112(4):A25.

20. Hennigan TW, Franks PJ, Hocken DB, Allen-Mersh TG. Rectal examination in general practice. BM] 1990;301(6750):478-480. https://doi.org/10.1136\%2Fbmj.301.6750.478

Accepted 9 March 2017. 